\title{
ANALYSIS OF THE EXTREMELY LOW FREQUENCY MAGNETIC FIELD EMISSION FROM LAPTOP COMPUTERS
}

\section{Darko Brodić}

University of Belgrade, Technical Faculty in Bor, Vojske Jugoslavije 12, 19210 Bor, Serbia $\bowtie$ dbrodic@tf.bor.ac.rs, +381 30 424 555)

\begin{abstract}
This study addresses the problem of magnetic field emission produced by the laptop computers. Although, the magnetic field is spread over the entire frequency spectrum, the most dangerous part of it to the laptop users is the frequency range from 50 to $500 \mathrm{~Hz}$, commonly called the extremely low frequency magnetic field. In this frequency region the magnetic field is characterized by high peak values. To examine the influence of laptop's magnetic field emission in the office, a specific experiment is proposed. It includes the measurement of the magnetic field at six laptop's positions, which are in close contact to its user. The results obtained from ten different laptop computers show the extremely high emission at some positions, which are dependent on the power dissipation or bad ergonomics. Eventually, the experiment extracts these dangerous positions of magnetic field emission and suggests possible solutions.
\end{abstract}

Keywords: classification, ELF, EMF, laptop, measurement.

C 2016 Polish Academy of Sciences. All rights reserved

\section{Introduction}

A static magnetic field can be produced by natural or man-made sources [1]. According to its characteristics, its source can be successfully determined. The naturally originated magnetic field is characterized as static or very slowly changing. On the contrary, the man-made magnetic field is distinguished by its level peaks. The origin of man-made magnetic field emission is related to the electrical power. Most of man-made sources emit Extremely Low Frequencies (ELF) of around $50 \mathrm{~Hz}$ or $60 \mathrm{~Hz}$. Still, the various types of research consider all magnetic field emissions below $300 \mathrm{~Hz}$ as ELF [2]. It is clear that ELF magnetic field emission is firmly associated with our modern civilization. Today, the laptop computers are widely used. If they create dangerous levels of magnetic field emission, then a large proportion of population can be exposed. Hence, research on this issue is important.

A typical definition of a laptop describes it as a portable personal computer, which is sufficiently light and small to be carried. Accordingly, it can be used at any location, which enables its user to travel with it. A laptop can be powered either by a battery or Alternating Current (AC) which makes its usage versatile. However, a laptop can be seen as an all-in-one design computer. It means that - unlike a desktop computer, where its processing unit, monitor, keyboard, mouse, speaker and battery are separated - in a laptop they are connected and sealed into one piece. Nevertheless, it assures its full functionality as in desktop computers. Furthermore, the use of a battery similarly to an Uninterruptible Power Supply (UPS) for desktop computers enables using a laptop in the absence of AC power supply for a quite period (typically $2-10$ hours). The aforementioned properties bring high versatility to the laptop users. Fig. 1 shows the inside of laptop all-in-one design. 


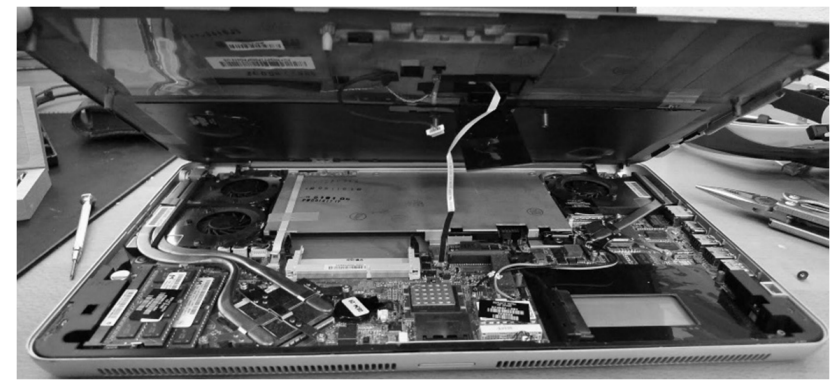

Fig. 1. The inside of laptop all-in-one design.

The expert scientific working group of the International Agency for Research on Cancer (IARC) has concluded that the earth's static magnetic field varies from $25 \mu \mathrm{T}$ at the equator to $65 \mu \mathrm{T}$ at the poles. This level of the magnetic field emission can be qualified as a natural one [1]. Many studies have been undertaken to examine destructive consequences of the ELF magnetic field exposure to the human health. The special attention has been focused on leukemia and tumors. In a monograph, IARC has concluded that the ELF magnetic field is possibly carcinogenic to humans. However, due to insufficient data, it could not be classified as a carcinogenic risk to humans [1]. Many analyses have shown that exposure to the ELF magnetic field higher than $0.4 \mu \mathrm{T}$ can double the risk of childhood leukemia [3]. However, no evidence has been found that link the higher ELF magnetic field exposure with tumours.

The risk of the ELF magnetic field exposure of laptop users has been analyzed in [3,4]. They proposed a reference limit level of the ELF magnetic field emission above which the environmental conditions can be unsafe for humans. The proposed critical limit level is equal to $0.3 \mu \mathrm{T}[3,4]$.

TCO stands for the Swedish organization "Confederation of Professional Employees" (in Swedish: Tjänstemännens Centralorganisation), which proposes different certification for computers and other office equipment. Its standards are accepted worldwide by manufacturers and users. The TCO standard in its TCO'05 version deals with the issue of laptop certification. It proposes a set of criteria which a tested product (i.e. a laptop) has to satisfy in order to be safe for the normal use. Hence, the aim of this document is to provide relevant test methods and criteria for the safe use of a product (see Fig. 9 in the Appendix for reference) [5]. Among the other criteria, TCO proposed the measurement methodology and the reference level of safe magnetic field exposure. The TCO standard divides the low frequency magnetic field into two bands: TCO band I and II. TCO band I includes the frequencies from $5 \mathrm{~Hz}$ to $2 \mathrm{kHz}$, whereas TCO band II deals with the frequencies from $2 \mathrm{kHz}$ to $400 \mathrm{kHz}$. The reference limits are 200 $\mathrm{nT}$ for the TCO band I and $25 \mathrm{nT}$ for the TCO band II [5], respectively. Although TCO band II has the stricter reference limit, it is much less likely to be violated because the main sources of magnetic field emission are much below this frequency range. Furthermore, the TCO standard recommends measuring the magnetic field at $0.30 \mathrm{~m}$ in the front of and around a laptop (see Fig. 9 in the Appendix for reference) [5]. From the manufacturer point of view, this recommendation seems reasonable. But, in real circumstances, it is pointless. Actually, the laptop users constantly type on the keyboard or click the touchpad. Hence, the user with his/her fingers and hands is constantly in close contact with the laptop body, which has no position recommended to be measured by the TCO standard. Also, if a laptop is used regularly and is in close contact with the user's body, then the user's health might be subjected to the negative effects $[4,6]$.

In this study, the issue of the ELF magnetic field emission by laptops is addressed. The magnetic field generated by 10 different laptops is measured. The laptops are tested in normal indoor operating conditions. Then, the measurement results are presented and compared 
according to the proposed risk standards. Also, some modification of working with a laptop is suggested in order to reduce the risk caused by the ELF magnetic field emission in the laptop's neighborhood.

The paper is organized in the following way. Section 2 explains the static magnetic field issue. Then, it describes the measuring devices and proposes the laptop's magnetic field measuring positions. Section 3 gives the measurement results of the experiment and discusses them. Section 4 contains conclusions.

\section{Materials and methods}

\subsection{Static magnetic field}

Each laptop represents an electronic system with many built-in electronic components. These electronic components need a current for the normal operation. Furthermore, the flow of the current $I$ through the electronic components induces a magnetic field. If the current $I$ is steady and constant in straight and uniform wire $C$, then the induced magnetic induction $\mathbf{B}(\mathbf{r})$ at the position $\mathbf{r}$ is calculated with the final equation of the Biot-Savart law:

$$
\mathbf{B}(\mathbf{r})=\frac{\mu_{0} I}{4 \pi} \int_{C} \frac{d \mathbf{l} \times \hat{\mathbf{r}}^{\prime}}{|\mathbf{r}|^{2}},
$$

where: $\mu_{0}$ is the magnetic permeability; $I$ is the current flowing through the wire $C ; \pi$ is the mathematical constant. The integral sums over the wire length $C$, the vector $d \mathbf{l}$ is the vector whose magnitude is the length of the differential element of the wire with its direction following that of the current $I$, and $\mathbf{r}^{\prime}$ is the displacement vector. It represents the displacement from the wire element $\mathbf{I}$ to the point $\mathbf{r}$, at which the field is computed, i.e. $\mathbf{r}^{\prime}=\mathbf{r}-\mathbf{I}$ [7]. The magnetic induction $\mathbf{B}(\mathbf{r})$ is characterized by its direction and magnitude. The direction can be decomposed into three-unit directional vectors in the directions along the Cartesian axes $x, y$, and $z$. This leads to the magnetic induction $\mathbf{B}(\mathbf{r})$ decomposed into the scalar components $B_{x}, B_{y}$ and $B_{z}$, which are measured in the directions of the Cartesian axes $x, y$ and $z$ :

$$
\mathbf{B}(\mathbf{r})=B_{x} \cdot \hat{\mathbf{x}}+B_{y} \cdot \hat{\mathbf{y}}+B_{z} \cdot \hat{\mathbf{z}} .
$$

The measurement devices usually register the scalar components of the magnetic induction $B_{x}, B_{y}$ and $B_{z}$ in a certain point. The magnitude of magnetic induction $|\mathbf{B}|$, usually marked as $B$, is calculated with these scalar components as [7]:

$$
B=\sqrt{\left(B_{x}^{2}+B_{y}^{2}+B_{z}^{2}\right)} .
$$

If $B$ is measured at different frequencies, then each of the measured values is marked $B_{i}$. The total Root Mean Square (RMS) $B_{\text {total }}$ is given as:

$$
B_{\text {total }}=\sqrt{\sum_{i=1}^{n} B_{i}^{2}}
$$

where $i=1, \ldots, n$, while $n$ represents the maximum number of frequencies at which the magnetic field $B$ is measured.

\subsection{Measuring device}

The origin of the magnetic field emitted by a laptop is its normal indoor operation A laptop is usually operated on an office desk. It means that the direction of the emitted magnetic field is at the top of the laptop only. Consequently, the magnetic field emission is prevented at its 
bottom due to the office desk position. Furthermore, the laptop user is in the close contact with his/her hands and fingers to the keyboard and/or touchpad. Such a close contact is determined by the zero distance to the laptop.

The measurement of magnetic field is performed by a professional AARONIA electromagnetic field spectrum analyzer Spectran NF-5035. The device measures the minimum, maximum, average and true Root Mean Square (RMS) magnetic induction from $1 \mathrm{pT}$ to $2 \mathrm{mT}$ in the full low frequency range between $1 \mathrm{~Hz}$ and $30 \mathrm{MHz}$. It has 6 typical measurement extents in the aforementioned frequency range. However, each user can define its own frequency measurement range contributing to its full customization. It is fully compliant to the extended ICNIRP range [8].

Furthermore, it has internal data storing capabilities of minimum $64 \mathrm{k}$ expandable to $1 \mathrm{MB}$. Downloading these data to a computer is performed via the USB connection. The obtained data are saved in .csv spreadsheet files, which are fully compliant with Microsoft Excel, OpenOffice Calc, etc.

Figure 2 shows the AARONIA spectrum analyzer Spectran NF-5035.

a)

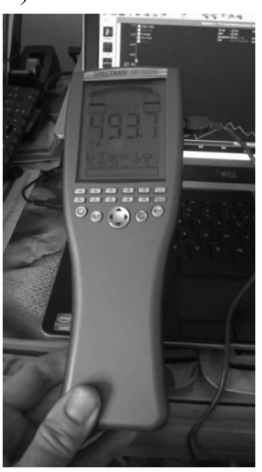

b)

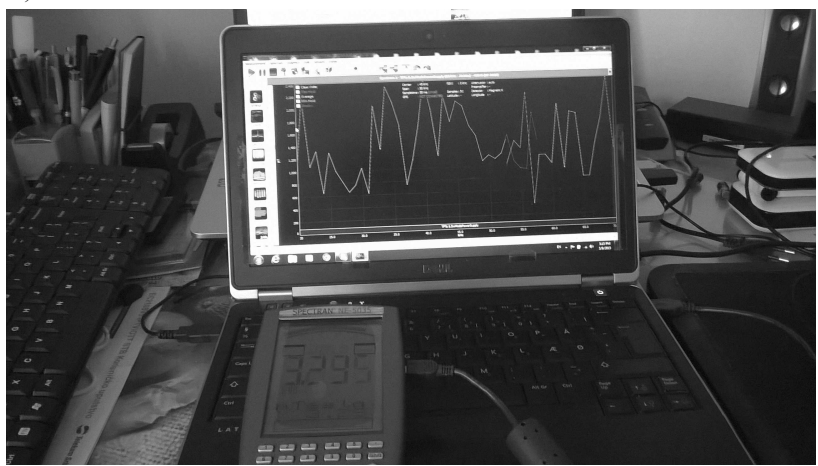

Fig. 2. The AARONIA spectrum analyzer Spectran NF-5035: a) the standalone unit; b) accompanying a computer for the real-time measurement.

\subsection{Laptop's measurement positions}

The magnetic field is measured at six different positions close to the laptop keyboard/touchpad instead of 9 proposed in $[9,10]$. Also, it is measured at the distance of $10 \mathrm{~cm}$ out of the laptop in the horizontal direction to the left or right from these six positions. At these 10 positions, the magnetic field is negligible. Hence, it is not important in the forthcoming analysis. Fig. 3 illustrates six magnetic field measuring positions at the laptop.

During normal office operation, the laptop is situated on the top of an office desk. Hence, the magnetic field emission below the desk is negligible. Furthermore, the user's hands and fingers are in a close contact with the keyboard and touchpad at the top of the laptop open body. That is why six positions at the top of the laptop are proposed for the measuring process.

The experiment included measuring 10 different laptops of different makes: L1, L2, .., L10. All measurements were taken under similar conditions with the AARONIA spectrum analyzer Spectran NF-5035. The measurements were performed with the described measuring device in the ELF slightly extended mode, i.e. from $50 \mathrm{~Hz}$ to $500 \mathrm{~Hz}$ with the step of $9 \mathrm{~Hz}$ and the sampling rate of $4 \mathrm{~s}$. Hence, $B_{\text {total }}$ according to the (4) is given as: 


$$
B_{\text {total }}=\sqrt{\sum_{i=1}^{51} B_{i}^{2}},
$$

where $i=1, \ldots, 51$, while $B_{1}=50 \mathrm{~Hz}, \ldots ., B_{51}=500 \mathrm{~Hz}$ with the step of $9 \mathrm{~Hz}$.

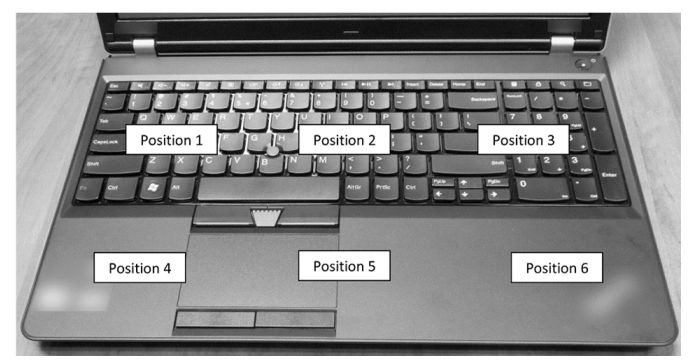

Fig. 3. The magnetic field measuring positions of a laptop in normal office operation.

\section{Results and discussion}

The magnetic field emission of a typical laptop in the frequency range between $50 \mathrm{~Hz}$ and $200 \mathrm{kHz}$ measured by the AARONIA NF-5035 device is presented in Fig. 4.

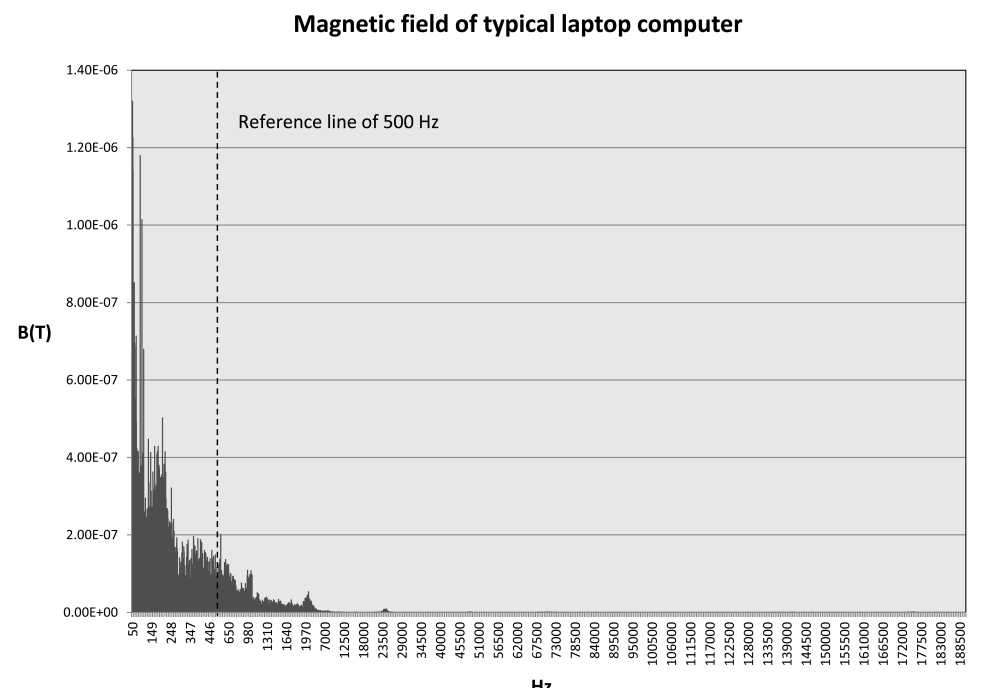

Fig. 4. A typical magnetic field emission of a laptop in the frequency range from $50 \mathrm{~Hz}$ to $200 \mathrm{kHz}$.

It shows three frequency measurement extents: (i) the extremely low frequency range (from $50 \mathrm{~Hz}$ to $500 \mathrm{~Hz}$ ), (ii) TCO band I, i.e. TCO1 (from $500 \mathrm{~Hz}$ to $2 \mathrm{kHz}$ ), and (iii) TCO band II, i.e. TCO2 (from $2 \mathrm{kHz}$ to $400 \mathrm{kHz}$ ). If we observe the results, it is clear that the magnetic field emission peaks are primarily in the area below $500 \mathrm{~Hz}$ (see the reference line in Fig. 4). Hence, the measurement of magnetic field below $500 \mathrm{~Hz}$ will be the object of concern in this study due to it being a cause of possible hazardous effects to the laptop user's health.

Figure 5 illustrates a typical distribution of the laptop's magnetic field emission from $50 \mathrm{~Hz}$ to $500 \mathrm{~Hz}$ at all six proposed measuring positions. In spite of the high peak of $1 \mu \mathrm{T}$ for around 
$50 \mathrm{~Hz}$, the positions 3 and 6 have the lowest level of the magnetic emission. Then, a slightly higher magnetic field emission is measured at the positions 1, 4 and 5 with the highest peaks of around $1.3 \mu \mathrm{T}$. Eventually, the highest level of the magnetic field is measured at the position 2 . It is characterized by an overwhelming level of the magnetic field emission with peaks of up to $3.5 \mu \mathrm{T}$.

a)

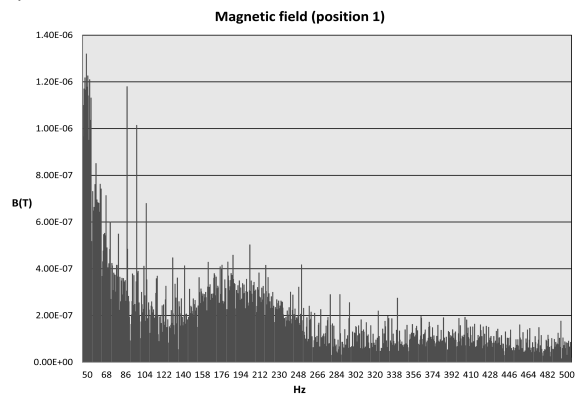

c)

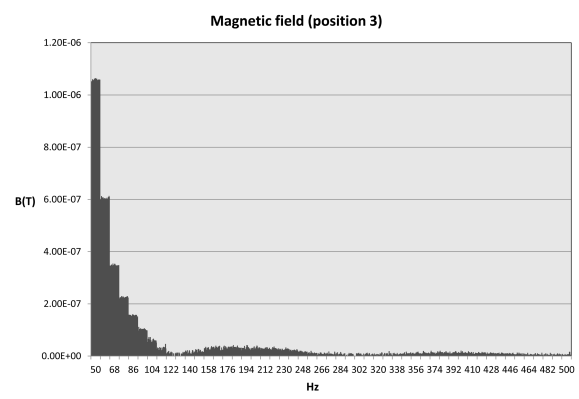

e)

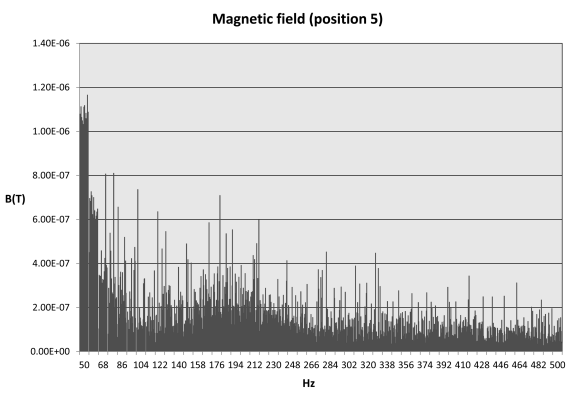

b)

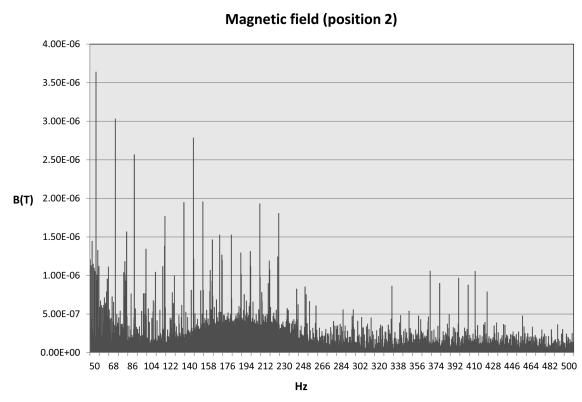

d)

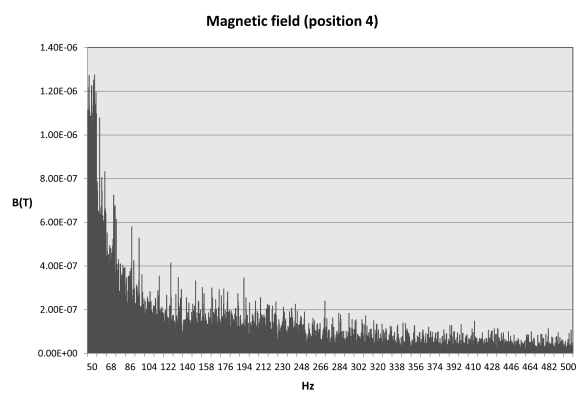

f)

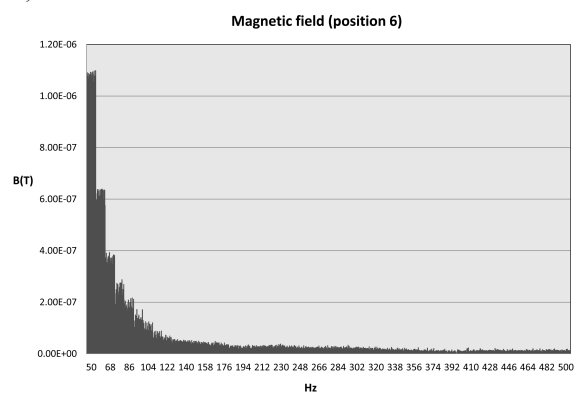

Fig. 5. A typical magnetic field emission in the frequency range from $50 \mathrm{~Hz}$ to $500 \mathrm{~Hz}$ : a) at the position 1; b) at the position 2 ; c) at the position 3 ; d) at the position 4 ; e) at the position 5 ; ) at the position 6 .

Furthermore, the measured magnetic field values for ten laptops with their minimum, maximum and average emission in the frequency range from $50 \mathrm{~Hz}$ to $500 \mathrm{~Hz}$ are given in Table 1.

The maximum values represent the peaks in the magnetic field. Sometimes, the peaks can be random. Hence, a more stable value for estimation of the magnetic field emission is represented by the average value. Still, the magnetic field emission of a laptop is a function of its built-in processor calculation power which can be characterized by the passmark value (CPU Passmark) [11], the Processor Total Power Dissipation (CPU TPD), and the Total Power 
Dissipation of the laptop (Max TD). Table 2 shows the aforementioned laptop characteristics of ten laptops.

Table 1. The measured magnetic field of ten tested laptops.

\begin{tabular}{|c|c|c|c|c|c|c|c|}
\hline Laptop & Type & Position 1 & Position 2 & Position 3 & Position 4 & Position 5 & Position 6 \\
\hline \multirow[t]{3}{*}{$\mathrm{L} 1$} & Minimum & $3.92 \mathrm{E}-07$ & 1.57E-07 & $1.05 \mathrm{E}-06$ & $9.96 \mathrm{E}-07$ & 0 & $1.02 E-06$ \\
\hline & Maximum & $1.32 \mathrm{E}-06$ & $3.64 \mathrm{E}-06$ & $1.06 \mathrm{E}-06$ & $1.28 \mathrm{E}-06$ & $1.17 \mathrm{E}-06$ & $1.10 \mathrm{E}-06$ \\
\hline & Average & $1.03 \mathrm{E}-06$ & $7.73 \mathrm{E}-07$ & $1.06 \mathrm{E}-06$ & $1.13 \mathrm{E}-06$ & $5.92 \mathrm{E}-07$ & $1.07 \mathrm{E}-06$ \\
\hline \multirow[t]{3}{*}{ L2 } & Minimum & $4.48 \mathrm{E}-09$ & $0.00 \mathrm{E}+00$ & $0.00 \mathrm{E}+00$ & $0.00 \mathrm{E}+00$ & $0.00 \mathrm{E}+00$ & $0.00 \mathrm{E}+00$ \\
\hline & Maximum & $1.26 \mathrm{E}-06$ & $1.16 \mathrm{E}-06$ & $1.20 \mathrm{E}-06$ & $1.08 \mathrm{E}-06$ & $1.08 \mathrm{E}-06$ & $1.16 \mathrm{E}-06$ \\
\hline & Average & $1.59 \mathrm{E}-07$ & $9.31 \mathrm{E}-08$ & 1.66E-07 & $3.72 \mathrm{E}-08$ & $3.76 \mathrm{E}-08$ & $4.32 \mathrm{E}-08$ \\
\hline \multirow[t]{3}{*}{ L3 } & Minimum & $0.00 \mathrm{E}+00$ & $2.11 \mathrm{E}-08$ & $1.71 \mathrm{E}-08$ & $0.00 \mathrm{E}+00$ & $0.00 \mathrm{E}+00$ & $0.00 E+00$ \\
\hline & Maximum & $1.31 \mathrm{E}-06$ & $1.32 \mathrm{E}-06$ & $1.20 \mathrm{E}-06$ & $1.08 \mathrm{E}-06$ & $1.07 \mathrm{E}-06$ & $1.08 \mathrm{E}-06$ \\
\hline & Average & $2.07 \mathrm{E}-07$ & $2.02 \mathrm{E}-07$ & $1.48 \mathrm{E}-07$ & $6.10 \mathrm{E}-08$ & $3.16 \mathrm{E}-08$ & $5.60 \mathrm{E}-08$ \\
\hline \multirow[t]{3}{*}{ L4 } & Minimum & $0.00 \mathrm{E}+00$ & $0.00 \mathrm{E}+00$ & $0.00 \mathrm{E}+00$ & $0.00 \mathrm{E}+00$ & $0.00 \mathrm{E}+00$ & $0.00 \mathrm{E}+00$ \\
\hline & Maximum & $1.34 \mathrm{E}-06$ & $1.06 \mathrm{E}-06$ & $1.06 \mathrm{E}-06$ & $1.07 \mathrm{E}-06$ & $1.09 \mathrm{E}-06$ & $1.34 \mathrm{E}-06$ \\
\hline & Average & $5.98 \mathrm{E}-08$ & $5.58 \mathrm{E}-08$ & $5.58 \mathrm{E}-08$ & $6.03 \mathrm{E}-08$ & $6.28 \mathrm{E}-08$ & $5.98 \mathrm{E}-08$ \\
\hline \multirow[t]{3}{*}{ L5 } & Minimum & $0.00 \mathrm{E}+00$ & $0.00 \mathrm{E}+00$ & $0.00 \mathrm{E}+00$ & $0.00 \mathrm{E}+00$ & $0.00 \mathrm{E}+00$ & $0.00 E+00$ \\
\hline & Maximum & $1.11 \mathrm{E}-06$ & $1.10 \mathrm{E}-06$ & $1.09 \mathrm{E}-06$ & $1.10 \mathrm{E}-06$ & $1.1 \mathrm{E}-06$ & $1.09 \mathrm{E}-06$ \\
\hline & Average & $8.92 \mathrm{E}-08$ & $4.62 \mathrm{E}-08$ & $5.90 \mathrm{E}-08$ & $5.78 \mathrm{E}-08$ & $3.74 \mathrm{E}-08$ & $5.24 \mathrm{E}-08$ \\
\hline \multirow[t]{3}{*}{ L6 } & Minimum & $0.00 \mathrm{E}+00$ & $0.00 \mathrm{E}+00$ & $0.00 \mathrm{E}+00$ & $0.00 \mathrm{E}+00$ & $0.00 \mathrm{E}+00$ & $0.00 E+00$ \\
\hline & Maximum & $1.16 \mathrm{E}-06$ & $1.11 \mathrm{E}-06$ & $1.10 \mathrm{E}-06$ & $1.09 \mathrm{E}-06$ & $1.13 \mathrm{E}-06$ & $1.10 \mathrm{E}-06$ \\
\hline & Average & $1.00 \mathrm{E}-07$ & 7.57E-08 & $7.22 \mathrm{E}-08$ & $3.79 \mathrm{E}-08$ & $2.85 \mathrm{E}-07$ & $4.48 \mathrm{E}-08$ \\
\hline \multirow[t]{3}{*}{$\mathrm{L} 7$} & Minimum & $0.00 E+00$ & $0.00 \mathrm{E}+00$ & $0.00 \mathrm{E}+00$ & $0.00 \mathrm{E}+00$ & $0.00 \mathrm{E}+00$ & $0.00 \mathrm{E}+00$ \\
\hline & Maximum & $4.09 \mathrm{E}-09$ & $1.06 \mathrm{E}-06$ & $1.06 \mathrm{E}-06$ & $1.51 \mathrm{E}-06$ & $5.89 \mathrm{E}-07$ & $1.08 \mathrm{E}-06$ \\
\hline & Average & $5.89 \mathrm{E}-10$ & $6.08 \mathrm{E}-08$ & $5.16 \mathrm{E}-08$ & $1.69 \mathrm{E}-07$ & $1.17 \mathrm{E}-07$ & $3.71 \mathrm{E}-08$ \\
\hline \multirow[t]{3}{*}{ L8 } & Minimum & $0.00 \mathrm{E}+00$ & $0.00 \mathrm{E}+00$ & $0.00 \mathrm{E}+00$ & $0.00 \mathrm{E}+00$ & $0.00 \mathrm{E}+00$ & $0.00 \mathrm{E}+00$ \\
\hline & Maximum & $1.74 \mathrm{E}-06$ & $1.19 \mathrm{E}-06$ & $1.10 \mathrm{E}-06$ & $1.47 \mathrm{E}-06$ & $1.12 \mathrm{E}-06$ & $1.10 \mathrm{E}-06$ \\
\hline & Average & $1.37 \mathrm{E}-07$ & $1.36 \mathrm{E}-07$ & $5.23 \mathrm{E}-08$ & $1.28 \mathrm{E}-07$ & $6.78 \mathrm{E}-08$ & $5.21 \mathrm{E}-08$ \\
\hline \multirow[t]{3}{*}{ L9 } & Minimum & $0.00 E+00$ & $0.00 \mathrm{E}+00$ & $0.00 \mathrm{E}+00$ & $0.00 \mathrm{E}+00$ & $0.00 \mathrm{E}+00$ & $0.00 \mathrm{E}+00$ \\
\hline & Maximum & $8.78 \mathrm{E}-06$ & 4.07E-07 & $1.03 \mathrm{E}-06$ & $2.02 \mathrm{E}-06$ & $6.64 \mathrm{E}-07$ & $1.04 \mathrm{E}-06$ \\
\hline & Average & $7.65 \mathrm{E}-07$ & 1.22E-07 & $8.25 \mathrm{E}-08$ & 1.54E-07 & $3.18 \mathrm{E}-08$ & $5.36 \mathrm{E}-08$ \\
\hline \multirow[t]{3}{*}{ L10 } & Minimum & $0.00 \mathrm{E}+00$ & $1.06 \mathrm{E}-08$ & $0.00 \mathrm{E}+00$ & $0.00 \mathrm{E}+00$ & $0.00 \mathrm{E}+00$ & $0.00 \mathrm{E}+00$ \\
\hline & Maximum & 1.11E-06 & $7.03 \mathrm{E}-06$ & $9.96 \mathrm{E}-08$ & $1.10 \mathrm{E}-06$ & $2.43 \mathrm{E}-07$ & $1.07 \mathrm{E}-06$ \\
\hline & Average & $5.96 \mathrm{E}-08$ & $7.68 \mathrm{E}-07$ & $4.39 \mathrm{E}-10$ & $7.56 \mathrm{E}-08$ & $9.8 \mathrm{E}-08$ & $5.38 \mathrm{E}-08$ \\
\hline
\end{tabular}

Table 2. A review of the typical characteristics of laptops.

\begin{tabular}{|c|c|c|c|}
\hline Laptop & CPU Passmark & CPU TD (W) & Maximum TD (W) \\
\hline L1 & 7493 & 45 & 120 \\
\hline L2 & 961 & 17 & 33 \\
\hline L3 & 2327 & 17 & 45 \\
\hline L4 & 782 & 18 & 65 \\
\hline L5 & 1687 & 25 & 65 \\
\hline L6 & 4007 & 35 & 90 \\
\hline L7 & 2113 & 35 & 90 \\
\hline L8 & 2646 & 35 & 80 \\
\hline L9 & 1157 & 35 & 80 \\
\hline L10 & 795 & 31 & 60 \\
\hline
\end{tabular}

The dependence of the laptop magnetic field emission on each of the characteristics like CPU passmark, CPU TPD and MAX TD can be evaluated by a correlation coefficient. The correlation coefficient $r$ expresses the strength and direction of a linear relationship between two variables $x$ and $y[12]$ : 


$$
r=\frac{\sum_{i}\left(x_{i}-\bar{x}\right)\left(y_{i}-\bar{y}\right)}{\sqrt{\sum_{i}\left(x_{i}-\bar{x}\right)^{2} \sum_{i}\left(y_{i}-\bar{y}\right)^{2}}},
$$

where: $\Sigma$ is the summation symbol; $x_{i}$ is the $x$ value for observation $i ; \bar{x}$ is the mean $x$ value; $y_{i}$ is the $y$ value for observation $i$; and $\bar{y}$ is the mean $y$ value. Fig. 6 shows the correlation coefficient $r$ between CPU passmark, CPU TPD and MAX TD and the level of the magnetic field at six measuring positions of a laptop, respectively.

\section{Correlation Coefficient}

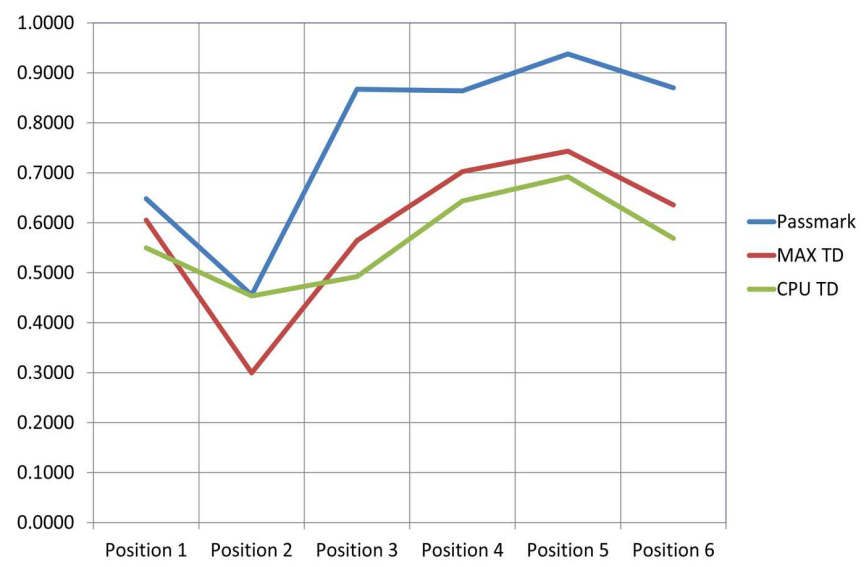

Fig. 6. The correlation coefficient $r$ between CPU passmark, CPU TPD and MAX TD and the level of magnetic field at six measuring positions of a laptop.

The correlation coefficient $r$ shows that the level of correlation between the laptop magnetic field emission at all six measured positions and the passmark is moderate to a very strong one. It reaches the values from 0.48 to 0.92 . At the positions 1 and 2 , the correlation is moderate to strong ( $r$ between 0.48 and 0.64 ), whereas at the positions 3-6 it is very strong ( $r$ between 0.87 and 0.92). Also, the values of correlation coefficient $r$ between the laptop magnetic field emission and CPU TD and MAX TD are between moderate and strong positive, respectively. Only at the position 2, the correlation between the laptop magnetic field emission and MAX TD is relatively weak positive, i.e. around 0.3. Furthermore, the correlation coefficient $r$ between the average magnetic field of all six measuring positions and three aforementioned laptop characteristics reaches even higher values. Table 3 shows the correlation coefficient $r$ values between the average magnetic field $B$ of a laptop and three laptop characteristics.

Table 3. The correlation coefficient $r$ values between the average magnetic field $B$ of a laptop and three laptop characteristics.

\begin{tabular}{|c|c|c|c|}
\hline Correlation Coefficient & CPU Passmark & CPU TPD & Max TD \\
\hline Average B & 0.8472 & 0.6490 & 0.6191 \\
\hline
\end{tabular}

Table 2 shows that the first laptop L1 has the highest values of the CPU passmark, CPU TPD and MAX TD. Hence, it should lead to a higher magnetic field emission. Furthermore, the laptop L2 has the lowest values of the CPU passmark, CPU TPD and MAX TD. Therefore, 
the lowest magnetic field emission is expected. In spite of that, bad ergonomics can be an additional correction factor.

Figure 7 shows the average values of magnetic field obtained for ten tested laptops.

a)

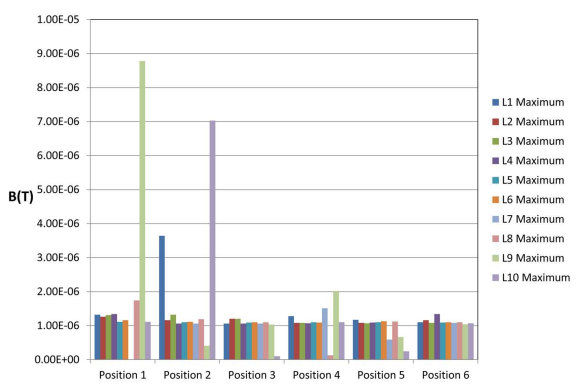

b)

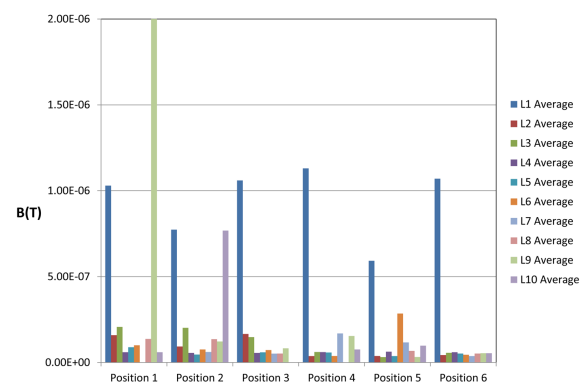

Fig. 7. The magnetic field emission of ten tested laptops: a) the maximum values; b) the average values.

The laptop L9 has the highest peak of the average magnetic field only at the position 2. Furthermore, it has quite acceptable values of the magnetic field at all other 5 positions. Because it has a low passmark value and a middle level of CPU TPD and Max TD values we can conclude about its bad ergonomic features. The laptop L1 has the highest passmark, CPU TPD and Max TD values. Hence, it has also high values of the magnetic field at all six positions. The laptop L10 has a noticeable peak only at the position 2. Because it has the lowest passmark value and middle level values of CPU TPD and Max TD, it also must have bad ergonomic features. The laptop L6 has a peak at the position 5. It presents a real case, because it has relatively high values of passmark, CPU TPD and Max TD. All other laptops seem to have acceptable values of the magnetic field emission.

Analysis of the measured magnetic field according to the safe reference level uses a typical level of $0.3 \mu \mathrm{T}$ as the safe reference [3, 4]. In these circumstances, the laptops L1, L9 and L10 have the emission exceeding the safe limit. To confirm this statement, a classification of peak and average values of magnetic field is created using the $k$-nearest neighbors $(k-\mathrm{NN})$ algorithm [13]. Fig. 8 shows a graphical representation of clusters for $K=4$.

a)

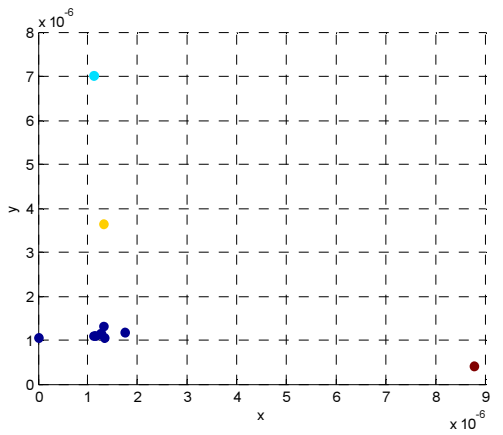

b)

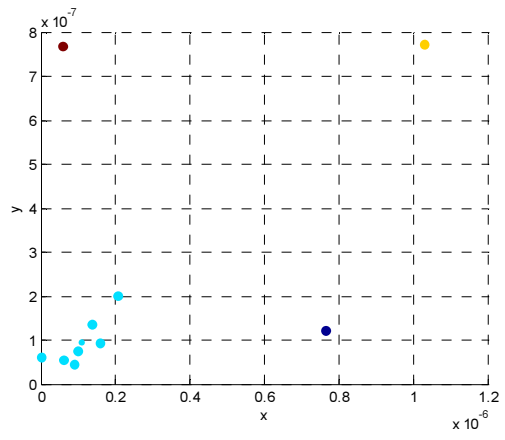

Fig. 8. The $K-\mathrm{NN}$ classification of measured magnetic field $(K=4)$ :

a) for maximum values: b) for average values.

Table 4 shows the classification into clusters with the $k$-NN algorithm. 
Table 4. The results of $k$-NN classification.

\begin{tabular}{|c|c|c|}
\hline Laptop & $\begin{array}{c}\text { Clustering } \\
\text { (Maximum values) }\end{array}$ & $\begin{array}{c}\text { Clustering } \\
\text { (Average values) }\end{array}$ \\
\hline $\mathrm{L} 1$ & 3 & 3 \\
\hline $\mathrm{L} 2$ & 2 & 1 \\
\hline $\mathrm{L} 3$ & 2 & 1 \\
\hline $\mathrm{L} 4$ & 2 & 1 \\
\hline $\mathrm{L} 5$ & 2 & 1 \\
\hline $\mathrm{L} 6$ & 2 & 1 \\
\hline $\mathrm{L} 7$ & 2 & 1 \\
\hline $\mathrm{L} 8$ & 2 & 1 \\
\hline $\mathrm{L} 9$ & 1 & 4 \\
\hline $\mathrm{L} 10$ & 4 & 2 \\
\hline
\end{tabular}

The classification with $k$-NN proves that the laptops L2, ..., L8 belong to the same cluster, which is considered safe for the laptop users. Three other laptops: L1, L9 and L10, are divided into three different clusters. The laptop L9 is classified in the most dangerous cluster. It has the highest peak and a high average level of magnetic field emission. It is a consequence of its bad ergonomic features. Then, the laptop L1 has a high magnetic field emission. However, it has also a high CPU processor power and a high dissipation. Hence, we can consider it as "normal" in this situation. The laptop L10 has a high peak and a relatively high average level of magnetic field emission. It is classified in a "less dangerous" cluster than that of the laptop L1. It is characterized by a very low passmark and a middle dissipation. Hence, its relatively dangerous level of magnetic field emission is a consequence of the bad ergonomic features.

Still, the long use of a laptop can expose the laptop users to the constantly high level of magnetic field. Hence, the recommendations to reduce the magnetic field exposure when using a laptop are: (i) if you use a laptop regularly, then make pauses, (ii) use an external keyboard whenever possible, (iii) use an external mouse regularly. However, analysis of the measured magnetic field according to the safe reference level should be performed. If we use a typical level of $0.3 \mu \mathrm{T}$ as the safe reference, then the laptops L1, L9 and L10 have the emission level exceeding the safe limit.

\section{Conclusions}

The paper addressed the issue related to the ELF magnetic field emission by laptop computers. The measurement of the magnetic field was performed by the AARONIA NF- 5035 device. The obtained results showed a clear dependence of the magnetic field value on three laptop characteristics: the CPU processor calculation power - passmark, the Processor Power Dissipation (CPU TD) and the laptop Total Power Dissipation (TPD). Furthermore, laptop ergonomics can be used as a corrective factor. The critical levels of the measured magnetic field were analyzed, too. Some laptops emitted magnetic field values exceeding the safe limits. The study pointed out the computer positions where the measured magnetic field emission was significant. This information could be exploited to use a portable computer safely and without a risk. Eventually, a few recommendations were suggested in order to use a laptop safely.

In future research, the orientation will be directed toward the creation of a laptop magnetic field emission model. To fulfil this task, measuring more positions between a laptop computer and its user, i.e. more than the six positions on the laptop computer, will be indispensable. 


\section{Acknowledgements}

This study was partly supported by the Ministry of Education, Science and Technological Development of the Republic of Serbia TR33037.

\section{Appendix}

TCO'05 proposed the measurement methodology of the magnetic field RMS value in two frequency ranges, i.e. in so-called band $1(50 \mathrm{~Hz}-2 \mathrm{kHz})$ and band II $(2 \mathrm{kHz}-400 \mathrm{kHz})$. TCO measuring positions are presented in Fig. 9.
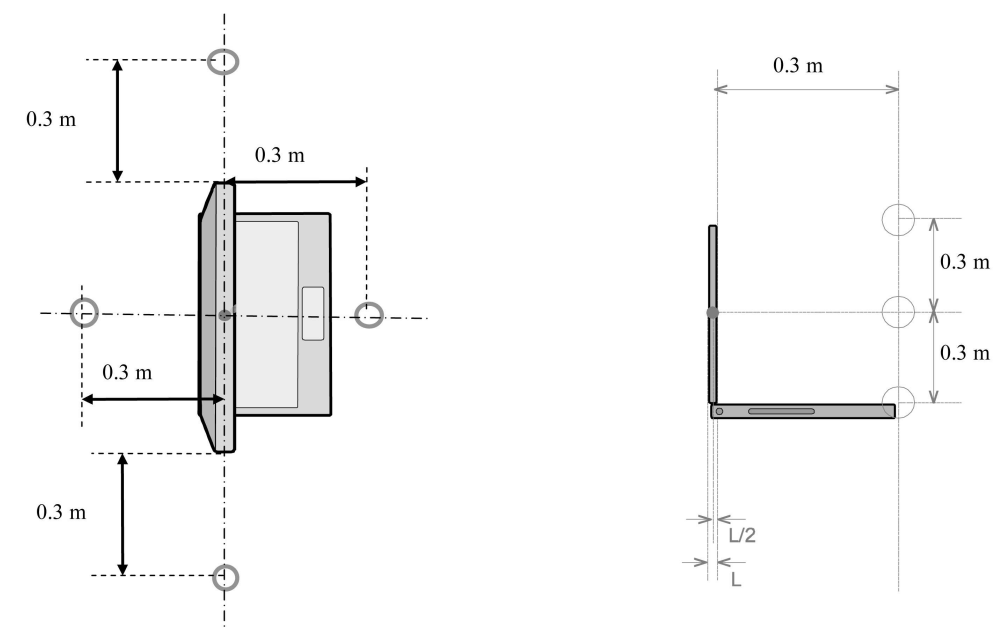

Fig. 9. The measurement geometry for the tested object [5].

From Fig. 9, it should be noted that the proposed measuring positions are quite different from the real positions at the laptop, which are in close contact with the hands and fingers. Hence, the results obtained according to the proposed measuring positions can be irrelevant to the health risk of the laptop users.

\section{References}

[1] International Agency for Research on Cancer (IARC). (2002). Monographs on the Evaluation of Carcinogenic Risks to Humans, Non-Ionizing Radiation, Part 1: Static and Extremely Low-Frequency (ELF) Electric and Magnetic Fields. Lyon, France: IARC Press, 80.

[2] National Radiological Protection Board (NRPB). (2001). ELF Electromagnetic Fields and the Risk of Cancer. Report of an Advisory Group on Non-ionizing Radiation (Doc. NRPB 12). Chilton, UK.

[3] Calvente, I., Fernandez, M.F., Villalba, J., Ilea, N. (2010). Exposure to electromagnetic fields and its relationship with childhood leukemia: A systematic review. Science of Total Environment, 408(16), 30622069.

[4] Bellieni, C.V., Pinto, I., Bogi, A., Zoppetti, N., Andreuccetti, D., Buonocore, G. (2012). Exposure to electromagnetic fields from laptop use of "laptop" computers. Archives of the Environmental and Occupational Health, 67(1), 31-36.

[5] TCO'05 standard. http://tcodevelopment.com/files/2013/04/TCO-Certified-Notebooks-4.0.pdf(2012). 
[6] Zoppetti, N., Andreuccetti, D., Bellieni, C., Bogi, A., Pinto, I. (2011). Evaluation and characterization of fetal exposures to low frequency magnetic fields generated by laptop computers. Progress in Biophysics \& Molecular Biology, 107(3), 456-463.

[7] Hayt, W., Buck, J. (2012). Engineering Electromagnetics. New York: McGraw-Hill, USA, 8th ed, 1-25.

[8] International Commision on Non-Ionizing Radiation Protection (ICNIRP). (1998). Guidelines for limiting exposure to time varying electric, magnetic and electromagnetic fields. Health Physics, 74, 494-522.

[9] Brodic, D., Amelio, A. (2015). Classification of the Extremely Low Frequency Magnetic Field Radiation Measurement from the Laptop Computers. Measurement Science Review, 15(4), 202-209.

[10] Brodic, D. (2015). Measurement of the Extremely Low Frequency Magnetic Field in the Laptop Neighborhood. Revista Facultad de Ingeniería, Universidad de Antioquia, 76, 39-45.

[11] https://www.cpubenchmark.net/

[12] Spiegel, M.R. (1992). Correlation Theory. Ch. 14 in Theory and Problems of Probability and Statistics. New York: McGraw-Hill, USA, 2nd ed, 294-323.

[13] Abbasifard, M.R., Ghahremani, B., Naderi, H. (2014). A Survey on Nearest Neighbor Search Methods. International Journal of Computer Applications, 95(25), 39-52. 